\title{
¿Cómo han gestionado los conservatorios de música españoles los procesos de enseñanza-aprendizaje durante el confinamiento del COVID-19?
}

How Spanish music conservatories managed pedagogy during the COVID-19 pandemic

Ramon Palau

ramon.palau@urv.cat

Facultad de Ciencias de la Educación y Psicología

Universidad Rovira y Virgili

Tarragona, España

ORCID: http://orcid.org/0000-0002-9843-3116

Jordi Mogas

jordi.mogas@urv.cat

Facultad de Ciencias de la Educación y Psicología

Universidad Rovira y Virgili

Tarragona, España

ORCID: http://orcid.org/0000-0003-3385-5534

María José Ucar

majoucar@hotmail.com

Conservatorios de Música de Castilla La Mancha

Castilla La Mancha, España

ORCID: http://orcid.org/0000-0002-8081-1078

doi: 10.7203/LEEME.46.18110

Recibido: 11-08-2020 Aceptado: 21-09-2020. Contacto y correspondencia: Ramon Palau, Universidad Rovira y Virgili, Facultad de Ciencias de la Educación y Psicología, Campus Sescelades, Carretera de Valls, s/n, C.P. 43007. Tarragona. España.

\section{Resumen}

Los conservatorios de música españoles han tenido que cerrar sus edificios con el objetivo de contener la propagación de la pandemia COVID-19. Los docentes de estos conservatorios han adoptado medidas para continuar con la enseñanza de forma remota utilizando recursos tecnológicos. El objetivo de esta investigación es analizar cómo los profesores de conservatorios españoles adaptaron los procesos de enseñanza y aprendizaje durante el cierre causado por la pandemia COVID-19 y revisar el potencial para cambiar el futuro de la educación musical. Este estudio se ha realizado mediante entrevistas a 36 docentes en activo de conservatorios de todas las comunidades autónomas de España. Los hallazgos muestran las dificultadas la enseñanza durante el confinamiento, la complejidad para virtualizar asignaturas de práctica instrumental, que esta se ha realizado en parte con recursos de los profesores, y que la competencia digital de los docentes genera comentarios diversos. Se evidencia la poca convicción sobre que los cambios realizados durante el confinamiento modifiquen aspectos tradicionales en la forma de enseñar ya que se muestran firmes defensores de la presencialidad.

Palabras clave: COVID-19; conservatorios de música; educación musical; competencia digital docente.

\section{Abstract}

Spanish music conservatories had to close their buildings with the aim of containing the spread of the COVID-19 pandemic. Teachers at these conservatories took steps to continue teaching remotely using technological resources. The objective of this research is to analyse how teachers of Spanish conservatories adapted the teaching and learning processes during the closure caused by the COVID-19 pandemic and review the potential to change the future of music education. This study has been carried out through interviews with 36 active teachers of conservatories from all the regions of Spain. The findings show the difficult teaching during lockdown, the complexity of virtualizing subjects of instrumental practice that this has been carried out in part, with teacher resources, and that the digital competence of teachers generates diverse comments. There is little conviction that changes made during confinement change traditional aspects of the way they teach. They are strong advocates of face-to-face teaching.

Key words: COVID-19; Spanish music conservatories; music education; teacher digital competence. 


\section{Introducción}

La crisis del COVID-19 se originó en Wuhan (Wu et al., 2020) y se expandió rápidamente por toda China y otras partes del mundo (Wickramasinghe et al., 2020). Llegó también al estado español y obligó al gobierno a imponer el confinamiento mediante el Real Decreto 463/2020 (Ministerio de la Presidencia, Relaciones con las Cortes y Memoria Democrática, 2020). Este confinamiento afectó a los centros educativos y entre ellos a los conservatorios de música. En este contexto, ha surgido un gran desafío para los conservatorios de música españoles, que se han visto obligados a buscar métodos alternativos para hacer la docencia.

La investigación en el campo de la educación durante la pandemia ha sido menos abundante que en otras disciplinas como medicina o economía. Según Sintema (2020), esto no se debe a que la educación no se ve directamente afectada por el efecto de la epidemia, sino porque hasta ahora, los estudios en educación rara vez han investigado los efectos de la enfermedad en los estudiantes de todo el mundo. En el campo de la educación musical, han aparecido menos investigaciones, aunque en el momento de la elaboración de este artículo se pueden ver algunas como Daubney y Fautley (2020), He (2020), o Kesendere et al. (2020), aunque ninguna de ellas sobre el territorio español. Esto justifica la relevancia de esta investigación, cuyo objetivo era analizar cómo los profesores de conservatorios españoles lograron adaptar los procesos de enseñanza y aprendizaje durante el cierre causado por la pandemia COVID-19 e indagar el potencial para cambiar el futuro de la educación musical.

\subsection{El confinamiento en la educación}

Las instituciones educativas han tenido que cambiar la modalidad de aprendizaje presencial al aprendizaje a distancia debido al cierre repentino de los centros educativos. Partiendo de que no había planes maduros y detallados para la educación en línea a gran escala en tiempos de emergencia (Zhang et al., 2020), las necesidades y los problemas no resueltos son extensos y van desde la falta de infraestructura, recursos, materiales y equipos por parte de las instituciones, administraciones y hogares de los confinados, hasta la falta de competencia digital por parte de los docentes (Burgess y Sievertsen, 2020; Crawford et al., 2020; Zhang et al., 2020).

En los últimos años, los avances tecnológicos han cambiado los comportamientos de aprendizaje de los alumnos y han reformado los métodos de enseñanza (Chen et al., 2016). De acuerdo con Crawford et al. (2020), la migración del aprendizaje tradicional o mixto a una estrategia de entrega totalmente virtual no se puede hacer de la noche a la mañana. Para comprender de manera efectiva los cambios que ocurren con el uso de las tecnologías de la información y la comunicación (TIC) y su uso por parte de los docentes, es esencial analizar el

@Ramon Palau, Jordi Mogas y María José Ucar. The content of this article is the sole responsibility of the authors. The Revista Electrónica de LEEME and Universitat de València are not liable for any legal actions that may arise involving the article's content. Revista Electrónica de LEEME - Lista Electrónica Europea de Música en la Educación-. http://ojs.uv.es/index/php/LEEME/index ISSN: 1575-9563. Editores: Universidad de Valencia y Jesús Tejada. Visibilidad de esta revista: SCOPUS, Emerging Sources Citation Index (Clarivate), EBSCO, CINDOC (CSIC), Citefactor, COPAC, Dialnet, DICE (CSIC), DOAJ, e-revistas (CSIC), EBSCO Premier, ERIH+, Gale Cengage Learning, IN-RECS, IRESIE, LATINDEX, MIAR, OCLC Worldcat, RESH, REDIB, RILM Core Journals, SUDOC, ULRICHS. Esta revista es de acceso libre mediante licencia Creative Commons 4.0 CC by. Política de archivado: etiqueta verde SHERPA-ROMEO. 

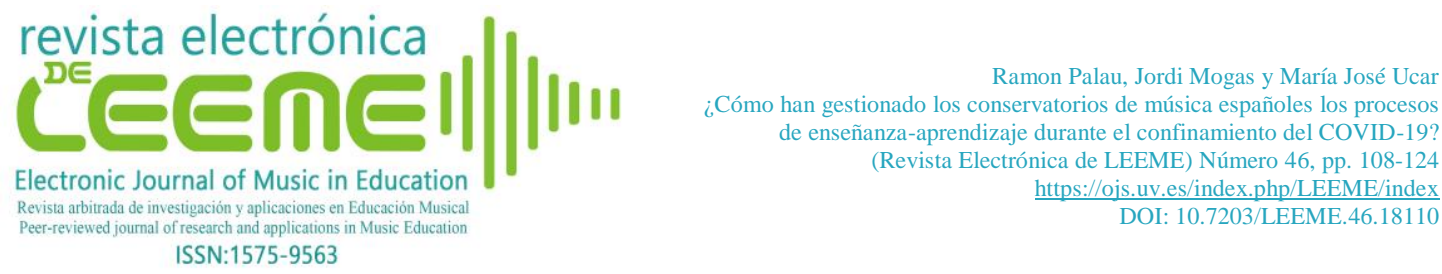

papel de la tecnología digital y los enfoques pedagógicos en las prácticas de enseñanza y aprendizaje (Prestridge et al., 2019).

Una de las claves del éxito de la tecnología es la selección de la herramienta que sustenta los procesos de enseñanza y aprendizaje y las competencias docentes (Aldunate y Nussbaum, 2013; Sailer et al., 2015). Bates (2020) sugiere varias recomendaciones para la implementación de modelos de formación online, que incluyen: obtener asesoramiento profesional y ayuda antes de comenzar, obtener la tecnología adecuada, organizarse, evitar largas conferencias, observar la carga de trabajo de los estudiantes, evitar conferencias en conjunto, y hacer lo mejor que pueda en las circunstancias.

El aprendizaje durante el confinamiento está sujeto a una separación entre alumno y docente. Está demostrado que la falta de asistencia a las clases produce el olvido de los contenidos previamente aprendidos (Sims, 2020). Sintema (2020) indica que los efectos adversos de la pandemia en la educación también pueden ser causados por la pérdida de horas de contacto entre los estudiantes, debido a la pérdida de interacción. Reducir la distancia transaccional mejora las experiencias de aprendizaje e impacta positivamente en los resultados del aprendizaje (Ekwunife-Orakwue y Teng, 2014). En este contexto, las decisiones tomadas sobre la interacción sincrónica o asincrónica entre docentes, estudiantes y sus familias son de particular importancia (Offir et al., 2008).

El efecto de la educación en línea depende de la capacidad y experiencia de enseñanza en línea de los docentes (Zhang et al., 2020). La competencia digital docente (CDD) se entiende como un conjunto de habilidades tecnológicas necesarias para el maestro actual. La CDD está determinada por las habilidades, actitudes y conocimientos requeridos por los educadores para apoyar aprendizaje de los estudiantes en el mundo digital de hoy (Hall et al., 2014). Krumsvik (2008; 2014) afirma que el uso básico de las TIC por parte de los docentes no es garantía de una práctica profesional competente, por lo que debe considerarse que la tecnología educativa no puede separarse de aspectos clave como el criterio pedagógico o el contexto educativo de aplicación.

\subsection{El confinamiento en la educación musical}

Existen pocas investigaciones sobre cómo se ha llevado a cabo la educación durante el período de confinamiento en instituciones musicales. Algunas de ellas analizan la posibilidad de la educación online (Daubney y Fautley, 2020; He, 2020; Kesendere et al., 2020). Aunque la educación musical, y especialmente en los conservatorios de música, es bastante conservadora en lo que respecta al uso de modalidades blended learning u online. Se han encontrado estudios anteriores al confinamiento, como Koutsoupidou (2014), Riley (2009), Orman y Whitaker (2010), Brändström et al. (2012), King et al. (2019), Cayari, (2018), Brook y Upitis, (2015),

\footnotetext{
@Ramon Palau, Jordi Mogas y María José Ucar. The content of this article is the sole responsibility of the authors. The Revista Electrónica de LEEME and Universitat de València are not liable for any legal actions that may arise involving the article's content. Revista Electrónica de LEEME - Lista Electrónica Europea de Música en la Educación-. http://ojs.uv.es/index/php/LEEME/index ISSN: 1575-9563. Editores: Universidad de Valencia y Jesús Tejada. Visibilidad de esta revista: SCOPUS, Emerging Sources Citation Index (Clarivate), EBSCO, CINDOC (CSIC), Citefactor, COPAC, Dialnet, DICE (CSIC), DOAJ, e-revistas (CSIC), EBSCO Premier, ERIH+, Gale Cengage Learning, IN-RECS, IRESIE, LATINDEX, MIAR, OCLC Worldcat, RESH, REDIB, RILM Core Journals, SUDOC, ULRICHS. Esta revista es de acceso libre mediante licencia Creative Commons 4.0 CC by. Política de archivado: etiqueta verde SHERPA-ROMEO.
} 


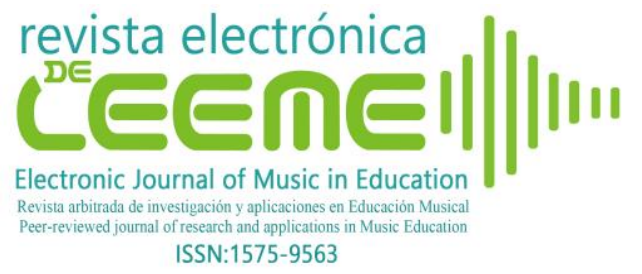

Waldron et al. (2018), van Gammeren y Szram (2019), Bayley y Waldron (2020) que analizan y muestran las posibilidades y los retos de estas modalidades en la enseñanza y aprendizaje de la música a partir de las nuevas posibilidades que ofrece la tecnología. Todas ellas valorando positivamente sus efectos y potencialidades, aunque matizando que la tecnología requiere de mejora o la importancia de las habilidades TIC y competencia digital de los docentes para su uso.

En el caso de los docentes de escuelas y conservatorios de música, la CDD ha sido poco estudiada, probablemente por su complejidad y por tener un modelo de formación diferente (Palau et al., 2019). Algunos de estos estudios muestran un uso limitado de la tecnología por parte de los profesores de los conservatorios, descubriendo que en general están poco motivados hacia la integración de las TIC en las enseñanzas musicales, alegando falta de tiempo y entrenamiento, a pesar de tener una actitud positiva hacia ellos (Belló, 2012; Colás y Hernández, 2014; Roig y García, 2014; Díez, 2018; Teachout, 2018).

\section{Método}

Presentamos en este apartado los detalles del estudio de corte cualitativo y enfoque fenomenológico, con posterior análisis descriptivo y de asociación. El propósito es realizar una comprensión profunda de la influencia de la pandemia de COVID-19 en el replanteamiento de los procesos y prácticas de enseñanza y aprendizaje (Flick et al., 2004), centrando la atención en los conservatorios de música españoles. Los datos se construyen en base a las experiencias de los participantes y su conocimiento tácito, lo que permite obtener información detallada de fenómenos específicos (Denzin y Lincoln, 2011; Silverman, 2006).

\subsection{Muestra}

El contexto de realización de este estudio ha sido el de los conservatorios de música españoles. La muestra ha sido elegida por conveniencia siguiendo criterios de heterogeneidad territorial y de edad. Han participado 36 docentes en activo de conservatorios de todas las comunidades autónomas de España, con la distribución detallada en la Figura 1. 

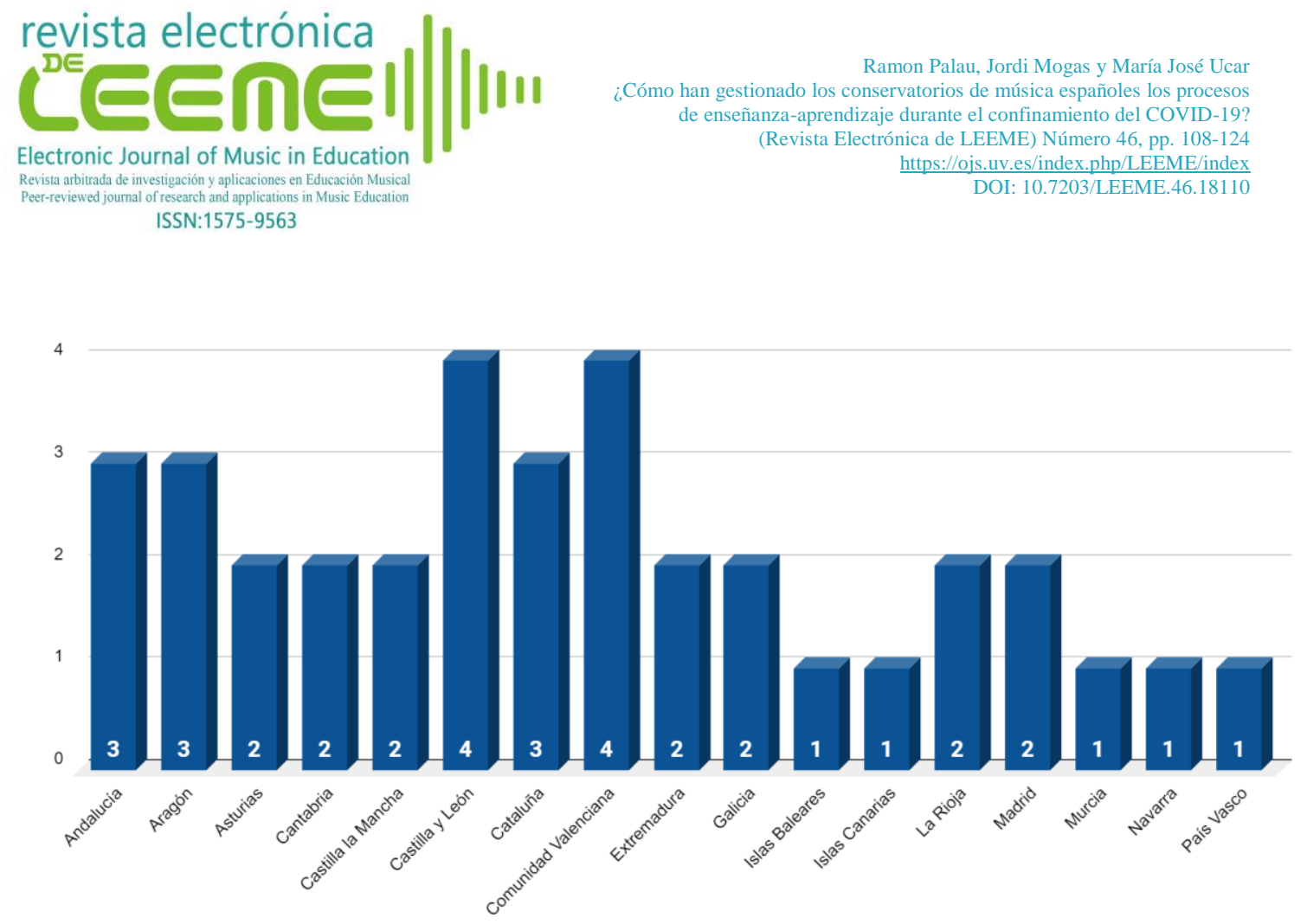

Fig. 1. Representación de los participantes según comunidades autónomas. Fuente: elaboración propia

Los docentes, que participaron voluntariamente, son 9 mujeres (25\%) y 27 hombres (75\%), de edades comprendidas en el rango 27 - 58 años (ver detalle en la Figura 2), y con una media de 44 años. En relación con el tipo de conservatorio, se cuentan 29 participantes que imparten docencia en conservatorios de tipo profesional (80\%) y 7 en conservatorios de tipo superior $(20 \%)$.

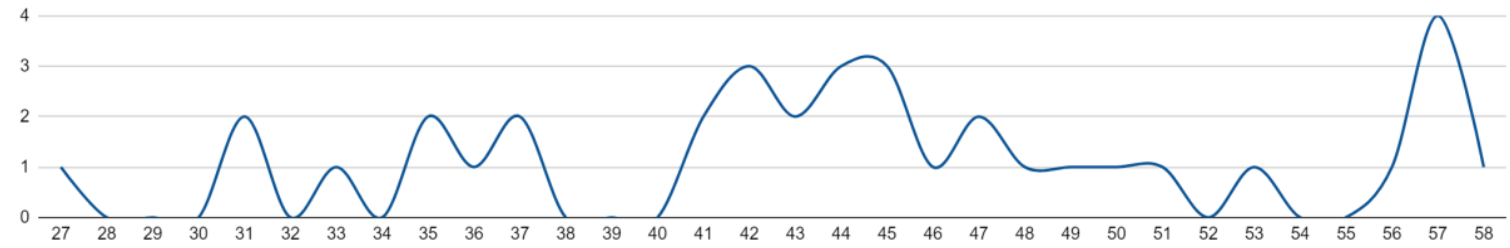

Fig. 2. Edades de los participantes. Fuente: elaboración propia

\subsection{Instrumento}

Para la recogida de datos se ha utilizado la entrevista estructurada como técnica e instrumento con la finalidad de obtener una comprensión profunda del problema que se investigaba mediante la documentación de las experiencias de los participantes (Kvale y Brinkmann, 2009; Minichiello et al., 1990). En concreto, se ha optado por la modalidad en línea para hacer llegar las preguntas de la entrevista a los diferentes participantes y que estos respondieran en un documento de texto a retornar una vez finalizada. Debida la situación social 


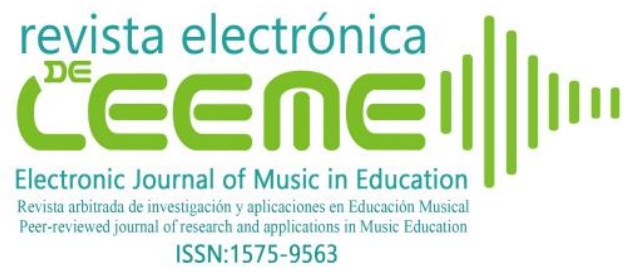

en la cual se enmarca esta investigación, las entrevistas tenían que realizarse forzosamente a distancia, y se optó por la realización de forma asincrónica y mediante redacción textual para permitir mayor nivel de libertad y reflexión a los participantes, pudiendo así ofrecer respuestas más elaboradas y profundas. Muchos autores han demostrado ya que las entrevistas en línea son un instrumento eficiente en la investigación cualitativa (Kitto y Barnett, 2007).

\subsection{Procedimiento}

Se cumplen las características de la investigación fenomenológica para diseños cualitativos que detalla Creswell (2012), habiendo realizado entrevistas a varios individuos que han experimentado una misma situación para comprender la esencia de la experiencia y describir el fenómeno vivido.

Los datos fueron recogidos con una toma de contacto con los conservatorios de música vía correo electrónico. Los participantes aceptaron voluntariamente ser entrevistados e informaron consentimiento para los usos investigativos de los datos recabados, siendo reconocida su anonimidad. Durante las semanas tercera y cuarta de la declaración del estado de alarma por parte del estado español se recibieron todas las respuestas.

Se ha utilizado el programa de análisis cualitativo de datos ATLAS.ti, versión 8.4.24. El procedimiento de análisis ha consistido en generar un documento matricial a partir de las respuestas de los participantes, el cual se ha cargado en el programa de análisis. En primer término, se ha procedido mediante un sistema de codificado abierto. La codificación en sentido amplio comprende los procedimientos de conceptualización, reducción, elaboración y vinculación de los datos (Strauss y Corbin, 2002). Para ello, se han añadido los códigos pertinentes en función de los datos recuperados. En total se han usado 16 códigos. Se han usado también dos memos, o notas de los investigadores, para guiar el proceso. En segundo término, y para facilitar el posterior análisis, se ha realizado una revisión mediante codificación axial depurando categorías y haciendo converger de forma gradual el análisis hacia aquellas categorías y códigos que aportan información más relevante para el presente estudio. Los datos obtenidos se han estructurado de acuerdo con las preguntas de investigación y se presentan en el apartado siguiente. 


\section{Resultados}

\subsection{Proceso de virtualización durante la situación de COVID-19}

\subsubsection{Adaptación metodológica de los docentes}

A partir de la declaración del estado de alarma, solamente se realizan de forma online actividades de refuerzo individuales. En ningún caso se informa de continuar con clases grupales. Asimismo, las asignaturas teóricas encuentran menor nivel de dificultad para pasar su programa a la docencia virtual, mientras que se remarcan dificultades para con sus asignaturas prácticas o instrumentales.

\footnotetext{
"Teniendo en cuenta que nuestros estudios se basan en la presencialidad, creo que los conservatorios se han visto muy afectados, por lo menos las clases en las que el instrumento es el principal protagonista. Habrá asignaturas teóricas, tipo historia de la música, que igual se pueden impartir telemáticamente, pero las clases de instrumento no son factibles en muchos casos" (Participante 11).
}

Mucho profesorado reconoce las dificultades propias de cambiar el sistema de docencia a online, pero lo aceptan y confirman que pese a ello pueden continuar con las clases en el nuevo formato. Algunos afirman haber cambiado su metodología docente para adaptarse a las clases online, aunque en realidad su esfuerzo se centra en utilizar recursos digitales para seguir con la metodología que usaban en las clases presenciales. Contados docentes reconocen un cambio metodológico decidido, siendo la siguiente la única respuesta contundente:

"Los cambios incorporados han sido muchos, en mis clases utilizaba la tecnología, pero como
herramienta de apoyo, no como medio para realizar mis clases, por lo que la metodología
también ha cambiado, tanto la forma de impartir las clases como de evaluarlas" (Participante $33)$.

Con todo, la gran mayoría de profesores entrevistados indican que no han cambiado la metodología de enseñanza en su paso a la modalidad virtual: "En mi caso, la metodología no ha supuesto prácticamente ningún cambio" (Participante 18), "La metodología es la misma, aunque tiene un carácter más informal" (Participante 16), "Cambios metodológicos no, sigo con mi programación y mi manera de dar clase, aunque no es lo mismo" (Participante 23), "La metodología de las clases no la he modificado, excepto, por supuesto, la característica de no ser presencial" (Participante 24).

\subsubsection{Recursos usados}

La totalidad de participantes informan sobre el uso de recursos propios, pudiéndose clasificar en dos grupos: uno que utiliza las herramientas propuestas desde la coordinación, 


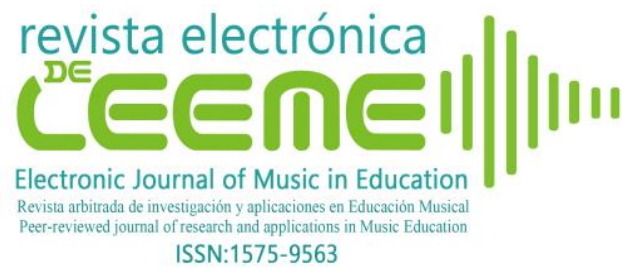

aunque con libertad para complementar con recursos adicionales, y otro donde cada profesor decide qué recursos usar.

"La Junta de Andalucía ha puesto a nuestra disposición todas las aplicaciones docentes disponibles, que son adecuadas para este cometido" (Participante 01).

"Cada profesor está actuando libremente, por lo que utilizamos las aplicaciones que consideramos necesarias y oportunas" (Participante 02).

Se trata siempre de aplicaciones para realizar videoconferencias (Skype, Zoom, FaceTime, Teams, Webex, Houseparty, Jitsi Meet, Discord y OneNote), para la comunicación textual (WhatsApp y correo electrónico), para la comunicación oral (llamadas de teléfono o llamadas de voz mediante aplicaciones), así como compartición audiovisual asíncrona (grabaciones de audio y vídeo). En casos más excepcionales, se mencionan otros recursos TIC para dar soporte a la enseñanza (YouTube o piano digital). Solo un caso menciona el uso de CentrosNet y otro afirma usar Moodle y una web personal.

"Utilizo un piano digital, así como otras herramientas que me permiten realizar ejemplos de forma rápida para que luego el alumno lo realice" (Participante 07).

Algún profesorado informa del uso de recursos físicos o de telecomunicación como elementos que ellos tienen que aportar:

"Los profesores hemos puesto nuestros recursos personales (teléfono, ordenador, instrumento, líneas de teléfono y wifi, software, adecuación de un espacio de la casa) al servicio de una asistencia docente online para la cual nos estamos teniendo que autoformar" (Participante 19).

\subsubsection{Problemas y limitaciones}

Los principales problemas han sido de carácter técnico, por dificultad de los alumnos a la hora de utilizar los recursos digitales necesarios: "problemas puntuales de acceso a las plataformas por parte de alumnos-padres" (Participante 01) o "la falta de conexiones a internet por falta de fibra en las áreas rurales" (Participante 08). Se evidencia especialmente la dificultad de atender diferentes necesidades de la práctica docente habitual en lo relativo a no poder dar soporte presencial a aspectos que lo requieren:

\footnotetext{
"Los aspectos concretos de la enseñanza presencial que no se pueden suplir con la enseñanza online, según mi opinión, a saber: (a) Corrección de postura del alumno, tanto del propio cuerpo del alumno como la colocación del instrumento. Esta cuestión, durante los primeros cursos de elemental es fundamental, y es muy difícil hacerlo online. (b) Interpretación de conjunto: resulta muy difícil hacer una interpretación a dúo, ya que existe un retardo en la línea de unos 0,5 segundos, lo que hace dificilísimo cuadrar rítmicamente una interpretación a dúo. De la misma manera, es dificilísimo marcar el pulso al alumno, pues ese retardo en la línea antes mencionado provoca serios problemas de conjunción rítmica. (c) Rectificación de lengüetas del alumno. (d) Corrección de pequeños desajustes en el instrumento del alumno" (Participante 01).
} 


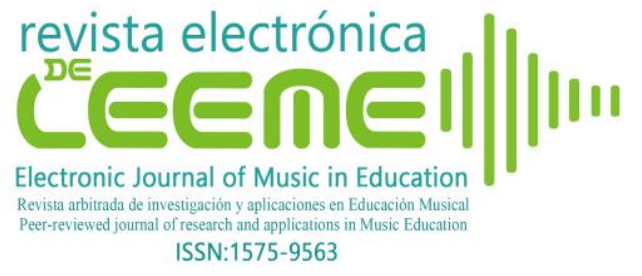

\subsubsection{Evaluación}

Tampoco hay consenso en los diferentes conservatorios en lo que refiere a la evaluación. Mientras algunos confirman haber suspendido cualquier tipo de proceso evaluativo, otros informan hacer seguimiento y evaluación online. Por parte del alumnado, la evaluación aparece como la principal preocupación. 16 de los 36 entrevistados reconocen que las calificaciones son un tema que preocupa a sus estudiantes.

\subsection{Competencia digital docente}

No existe consenso en las respuestas a la pregunta sobre si el nivel de competencia digital docente (CDD) de los docentes de cada conservatorio ha sido suficiente para afrontar el reto. En la tabla 1 se presentan las frecuencias en el sentido de la valoración de los participantes por rango de edad, pero estos datos no se pueden interpretar en términos absolutos, sino que se deben leer con los matices que se remarcan a continuación.

Tabla 1. Sentido de las respuestas sobre el nivel de CDD de los docentes del conservatorio es suficiente.

\begin{tabular}{lccccc}
\hline & & \multicolumn{3}{c}{ REPRESENTACIÓN } & \\
\cline { 3 - 5 } & & POSITIVA & NEGATIVA & DUBITATIVA & Total \\
\hline Rango de edad & $27-39$ & 2 & 2 & 5 & 9 \\
del entrevistado & $40-49$ & 3 & 3 & 9 & 15 \\
& $50-59$ & 4 & 6 & 2 & 12 \\
Total & 9 & 11 & 16 & 36 \\
\hline
\end{tabular}

Fuente: Elaboración propia

Varios de los participantes que responden afirmativamente puntualizan que el sentido de la respuesta se fundamenta en las decisiones tomadas a nivel de centro. Es decir, se declinan por afirmar que existe una CDD suficiente entre los profesores de su centro, pero dados unos criterios específicos no objetivables o comparables a una valoración general.

"Con las medidas adoptadas sí. Si se hubiesen tomado otro tipo de medidas de seguimiento casi seguro que no" (Participante 13).

Se entiende que son respuestas afirmativas que podrían haber sido también negativas en la mitad de los casos, aproximándose así a la opinión negativa de 11 participantes.

"Creo que hay parte de la plantilla capacitada para trabajar con nuevas tecnologías y suficientemente competente como para aprender y adaptarse rápidamente, pero el grueso del claustro tiene carencias enormes. Hasta tal punto que algunas asignaturas no se están impartiendo" (Participante 12).

"Rotundamente no. Creo que el nivel de Competencia Digital Docente por norma general en los conservatorios es bajo, ya que son unas enseñanzas que muy tradicionales en las que 


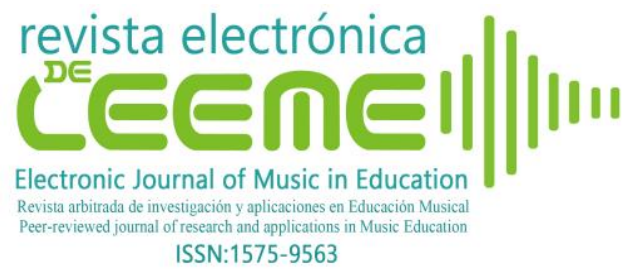

cuesta salir y buscar diferentes opciones a las clases presenciales del 'tú a tú'" (Participante 25).

Por último, hay profesorado que se muestra dubitativo por dos motivos principales. Uno, porque cree que no puede opinar (tres casos); otro, porque cree que hay factores que afectan la CDD, como podrían ser el tipo de docencia que se debe impartir (dos casos) y, muy especialmente, porque considera que existe una brecha de edad: el profesorado joven tiene mayor CDD y los mayores sufren mayor dificultad con el uso de las tecnologías digitales (once casos).

\footnotetext{
"Por norma general, intuyo que el profesorado más joven es más competente que el de más edad" (Participante 10).

"Me consta que el profesorado de mayor edad no está llevando las clases a cabo, supongo que por desconocimiento de las tecnologías" (Participante 25).
}

De los once casos dubitativos por razón de edad (i.e. no se pronuncian en un sentido porque creen que con distintas edades la respuesta varía), encontramos la siguiente representación:

- 3 de los 9 en el rango de edad 27-39 (33\%).

- 8 de los 15 en el rango 40-49 (53\%).

- Ninguno de los 12 en el rango 50-59(0\%).

Esta situación es vista en todos los casos como una oportunidad en la formación para mejor capacitación en lo que respecta a CDD. Ningún entrevistado reconoce haber realizado formación específica con elevada dedicación, pero la mayoría remarcan que la posibilidad u oportunidad de formación será una consecuencia de la pandemia.

\footnotetext{
"Cambiará totalmente la percepción, a partir de ahora, muchos docentes seremos conscientes de lo atrasados que estamos tecnológicamente. A partir de ahora entenderemos que las tecnologías pueden ser nuestras aliadas" (Participante 02).
}

\subsection{Efectos sobre las prácticas educativas futuras}

Prácticamente la totalidad del profesorado entrevistado (el 91,7\%) afirma que a partir de ahora no existirán cambios. Por una parte, encontramos docentes firmes defensores de la presencialidad en el aprendizaje musical. Todos coinciden en remarcar que se trata de un tipo de enseñanza tradicionalmente presencial, donde el contacto directo con el alumno es esencial. A menudo, apuntan que valoran positivamente las nuevas tecnologías, pero como complemento y no como substituto de la presencialidad.

"No creo que las enseñanzas de música estén diseñadas para ser impartidas de manera telemática. Considero que esta situación excepcional y las medidas que se han adoptado son un parche para intentar sobrellevar esta situación, pero de ningún modo esta forma de impartir 


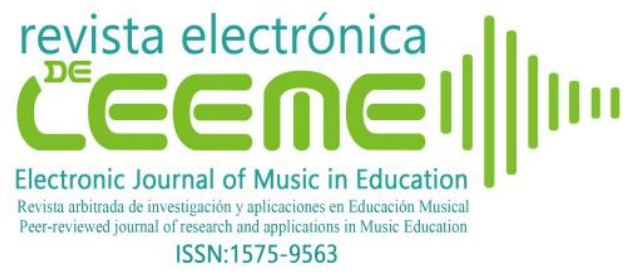

clases puede suplir las clases presenciales tan necesarias en unas enseñanzas de este tipo" (Participante 06).

Por otra parte, limitadas respuestas prevén algún cambio hacia una virtualización más estructural, pero en cualquier caso de forma secundaria, aclarando que la pandemia no modificará los aspectos tradicionales en la forma de enseñar y que en ningún caso podrá servir para las asignaturas instrumentales y grupales. Algunos se centran en la oportunidad de pedir al centro mejores recursos, como por ejemplo la adopción de la aplicación CentrosNet para la comunicación, o bien conexión wifi en el conservatorio.

"[...] ha abierto una posibilidad para poder digitalizar ciertas asignaturas cuyas clases presenciales no son esenciales (asignaturas teóricas) y la posibilidad que ahora no existía de poder atender al alumnado de manera virtual en situaciones excepcionales” (Participante 22).

En sentido contrario, solamente tres de los 36 participantes prevén o reclaman modernización en clave de nuevas tecnologías después de la pandemia. A destacar:

\begin{abstract}
"La metodología empleada hasta ahora en las enseñanzas musicales se encontraba obsoleta ya que estaba basada en el mismo modelo que se utilizaba hace 50 años. En mi opinión, la nueva situación va a acelerar el cambio hacia un modelo educativo musical más adaptado a los nuevos tiempos, con más presencia de recursos on-line y restando importancia a los contenidos puramente teóricos y memorísticos, dando una dimensión práctica a los conocimientos adquiridos, que en muchas ocasiones quedaban en ideas inconexas e inaplicables" (Participante 17).
\end{abstract}

\title{
4. Discusión y conclusiones
}

El seguimiento de la enseñanza y aprendizaje en los conservatorios de música españoles durante el confinamiento ha evidenciado dificultades, de la misma forma que se ha evidenciado en otros países y contextos (Zhang et al., 2020). Entre estas dificultades, una de las principales ha estado el proceso de virtualización tal y como señalan Crawford et al. (2020) cuando indican que ésta no se puede hacer de la noche a la mañana, o Bates (2020) cuando remarca que esta transformación educativa debe seguir ciertas pautas de diseño. Los docentes explican que básicamente se han centrado en buscar recursos para seguir con sus planes y enfoques existentes. En este sentido, Prestridge et al. (2019) y Crawford et al. (2020) explican que para hacer un cambio se requiere un análisis del papel de la tecnología, los enfoques pedagógicos y habilidades para diseñar y ofrecer educación en línea.

El profesorado entrevistado informa que las asignaturas teóricas se han virtualizado con más facilidad, mientras que las instrumentales requieren de videoconferencias o grabación de videos y la modalidad online no resulta apropiada. Esto se alinea con las observaciones de Offir et al. (2008) sobre la importancia en la toma de decisiones sobre las interacciones sincrónicas o asincrónica entre docentes y discentes.

@Ramon Palau, Jordi Mogas y María José Ucar. The content of this article is the sole responsibility of the authors. The Revista Electrónica de LEEME and Universitat de València are not liable for any legal actions that may arise involving the article's content. Revista Electrónica de LEEME - Lista Electrónica Europea de Música en la Educación-. http://ojs.uv.es/index/php/LEEME/index ISSN: 1575-9563. Editores: Universidad de Valencia y Jesús Tejada. Visibilidad de esta revista: SCOPUS, Emerging Sources Citation Index (Clarivate), EBSCO, CINDOC (CSIC), Citefactor, COPAC, Dialnet, DICE (CSIC), DOAJ, e-revistas (CSIC), EBSCO Premier, ERIH+, Gale Cengage Learning, IN-RECS, IRESIE, LATINDEX, MIAR, OCLC Worldcat, RESH, REDIB, RILM Core Journals, SUDOC, ULRICHS. Esta revista es de acceso libre mediante licencia Creative Commons 4.0 CC by. Política de archivado: etiqueta verde SHERPA-ROMEO. 


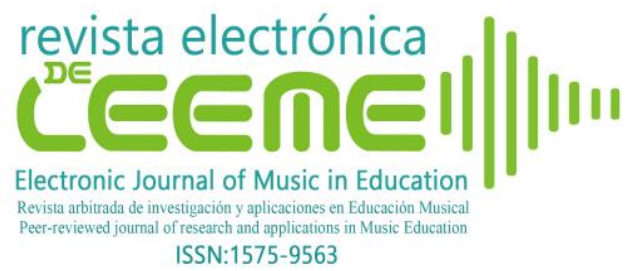

Aldunate y Nussbaum (2013) y Sailer et al. (2015) defienden que una de las claves del éxito de la tecnología es la selección de la herramienta. En este trabajo se pone de relieve que algunas instituciones han determinado unas directrices y sugerido recursos determinados, mientras que en otros conservatorios los docentes han recurrido a recursos que han encontrado por su cuenta, o que conocían previamente. En general, se puede afirmar que este aspecto fundamental no se ha abordado con la máxima eficiencia deseable desde un punto de vista estratégico.

La competencia digital docente (CDD) ha sido valora como suficiente con matices y opiniones, especialmente acotando a la situación de confinamiento y sin valorar que una digitalización generalizada pondría de relieve muchas más carencias. Varios participantes valoran que hay diversidad de niveles entre el profesorado, especialmente en lo que refiere a la edad. En esta línea, Palau et al. (2019) observaron una autopercepción similar a la media de docentes fuera de las enseñanzas musicales, en contra de otros estudios (Belló, 2012; Colás y Hernández, 2014; Roig y García, 2014; Díez, 2018; Teachout, 2018). Los entrevistados en el presente trabajo, valoran esta situación como una oportunidad en la formación para mejor capacitación en lo que respecta a CDD.

Sobre cómo este proceso producirá cambio en el futuro, los entrevistados en general se han mostrado firmes defensores de la presencialidad en el aprendizaje musical, del mismo modo que Ekwunife-Orakwue y Teng (2014). Existen resultados positivos de experiencias sobre virtualización, blended learning y educación en línea en la educación musical (Koutsoupidou, 2014; Crawford, 2017; King et al., 2019; Cayari, 2018; Brook y Upitis; 2015), los cuales cabría presentar a los docentes, que no los tienen presentes, aunque ya valoran positivamente nuevos enfoques pedagógicos.

Los resultados muestran la poca convicción sobre que los cambios realizados durante el confinamiento modifiquen los aspectos tradicionales en la forma de enseñar música. Sobre todo, se encuentra dificultad en las asignaturas instrumentales, ya sean individuales o colectivas, que seguirán como anteriormente. Contrariamente, autores como Chen et al. (2016) o Yuen y Will (2008), demostraron que los avances tecnológicos han cambiado los comportamientos de aprendizaje de los alumnos y han reformado los métodos de enseñanza y el uso pedagógico exitoso de la tecnología también depende de las actitudes de los docentes. Una explicación a ello puede ser la visión de las TIC que tienen los profesores de conservatorios españoles (Belló, 2012; Colás y Hermnández, 2014; Roig y García, 2014; Díez, 2018), o que antes de ofrecer educación en línea es necesario un análisis del papel de la tecnología y los enfoques pedagógicos (Crawford et al., 2020). En cambio, lo que piden los docentes es la mejora de los recursos tecnológicos como la conexión a internet o disponer de alguna plataforma institucional.

Hasta el momento no se han encontrado investigaciones sobre los conservatorios durante el confinamiento que permitan contrastar los resultados, lo que se dibuja como principal

@Ramon Palau, Jordi Mogas y María José Ucar. The content of this article is the sole responsibility of the authors. The Revista Electrónica de LEEME and Universitat de València are not liable for any legal actions that may arise involving the article's content. Revista Electrónica de LEEME - Lista Electrónica Europea de Música en la Educación-. http://ojs.uv.es/index/php/LEEME/index ISSN: 1575-9563. Editores: Universidad de Valencia y Jesús Tejada. Visibilidad de esta revista: SCOPUS, Emerging Sources Citation Index (Clarivate), EBSCO, CINDOC (CSIC), Citefactor, COPAC, Dialnet, DICE (CSIC), DOAJ, e-revistas (CSIC), EBSCO Premier, ERIH+, Gale Cengage Learning, IN-RECS, IRESIE, LATINDEX, MIAR, OCLC Worldcat, RESH, REDIB, RILM Core Journals, SUDOC, ULRICHS. Esta revista es de acceso libre mediante licencia Creative Commons 4.0 CC by. Política de archivado: etiqueta verde SHERPA-ROMEO. 


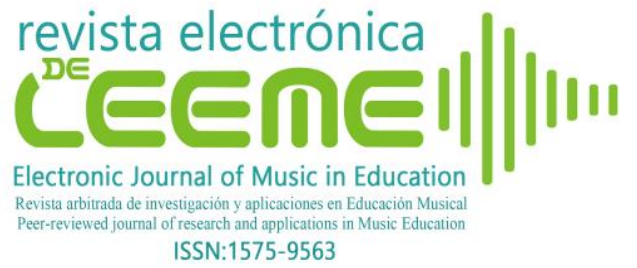

limitación de este estudio y plantea las consecuentes líneas futuras de investigación. Además, se extraen las siguientes implicaciones para futuras acciones:

- Se debe hacer una profunda reflexión sobre las percepciones y visiones del profesorado de conservatorios españoles sobre cómo la tecnología o la virtualización podría ayudar a la mejora y flexibilidad de la formación ofrecida.

- Debe plantearse cómo mejorar la CDD del profesorado de música de conservatorio y los recursos para poderla desarrollar y aplicar, como las conexiones a Internet, plataformas institucionales y dispositivos.

- Los conservatorios deben plantearse planificar un posible plan de virtualización en caso de otro bloqueo, previendo la formación docente requerida, estrategias pedagógicas para los diferentes recursos digitales y plataformas.

- Conviene estudiar en mayor detalle las diferencias de cómo afecta la situación sobrevenida por el COVID-19 entre centros elementales, profesionales y superiores.

\section{Referencias}

Aldunate, R. y Nussbaum, M. (2013). Teacher adoption of technology. Computers in Human Behavior, 29(3), 519-524. doi:10.1016/j.chb.2012.10.017

Bates, A.W. (2020). Advice to those about to teach online because of the corona-virus. Recuperado de: https://www.tonybates.ca/2020/03/09/advice-to-those-about-to-teachonline-because-of-the-corona-virus/

Bayley, J.G. y Waldron, J. (2020). "It's never too late": Adult students and music learning in one online and offline convergent community music school. International Journal of Music Education, 38(1), 36-51. doi:10.1177/0255761419861441

Belló, J.M. (2012). Las Tecnologías de la Información y Comunicación en los conservatorios de Música de Galicia: análisis de los usos, actitudes y formación del profesorado. [Tesis doctoral]. Universidad de A Coruña.

Brändström, S., Wiklund, C. y Lundström, E. (2012) Developing distance music education in Arctic Scandinavia: electric guitar teaching and master classes. Music Education Research, 14(4), 448-456. doi:10.1080/14613808.2012.703173

Brook, J. y Upitis, R. (2015). Can an online tool support contemporary independent music teaching and learning? Music Education Research, 17(1), 34-47. doi:10.1080/14613808.2014.969217 
Burgess, S. y Sievertsen, H.H. (2020). Schools, skills, and learning: The impact of COVID-19 on education. Londres: VoxEu.

Cayari, C. (2018). Connecting music education and virtual performance practices from YouTube. Music Education Research, 20(3), 360-376. doi.:10.1080/14613808.2017.1383374

Chen, N.S., Cheng, I.L. y Chew, S.W. (2016). Evolution is not enough: Revolutionizing current learning environments to smart learning environments. International Journal of Artificial Intelligence in Education, 26(2), 561-581. doi:10.1007/s40593-016-0108-x

Colás, P. y Portero, G.H. (2014). Incidencia de la Formación del Profesorado en sus creencias sobre el valor de las TIC en la enseñanza de la Música. Educatio Siglo XXI, 32, 51-74. doi: $10.6018 / \mathrm{j} / 210981$

Crawford, J., Butler-Henderson, K., Rudolph, J., y Glowatz, M. (2020). COVID-19: 20 Countries' Higher Education Intra-Period Digital Pedagogy Responses. Journal of Applied Teaching and Learning, 3(1), 9-28. doi:10.37074/jalt.2020.3.1.7

Crawford, R. (2017). Rethinking teaching and learning pedagogy for education in the twentyfirst century: blended learning in music education, Music Education Research, 19(2), 195-213. doi:10.1080/14613808.2016.1202223

Creswell, J.W. (2007). Qualitative Inquiry and Research Design: Choosing Among Five Approaches (2nd ed.). California: SAGE Publications, Inc.

Daubney, A. y Fautley, M. (2020). Editorial Research: Music education in a time of pandemic. British Journal of Music Education, 37(2), 107-114. doi:10.1017/S0265051720000133

Denzin, N.K. y Lincoln, Y.S. (2011). Introduction: The Discipline and Practice of Qualitative Research. En N.K. Denzin e Y.S. Lincoln (Eds.), The SAGE Handbook of Qualitative Research (4th ed., pp.1-19). California: SAGE Publications, Inc.

Díez, N. (2018). La formación en TIC de los pedagogos de música. Análisis de la situación en las Enseñanzas Superiores de Música. Revista Electrónica de LEEME, 42, 31-51. doi:10.7203/leeme.42.13067

Ekwunife-Orakwue, K.C.V. y Teng, T.L. (2014). The impact of transactional distance dialogic interactions on student learning outcomes in online and blended environments. Computers \& Education, 78, 414-427. doi:10.1016/j.compedu.2014.06.011

Flick, U., von Kardorff, E. y Steinke, I. (Eds.). (2004). A Companion to Qualitative Research. California: SAGE Publications Ltd.

@Ramon Palau, Jordi Mogas y María José Ucar. The content of this article is the sole responsibility of the authors. The Revista Electrónica de LEEME and Universitat de València are not liable for any legal actions that may arise involving the article's content. Revista Electrónica de LEEME - Lista Electrónica Europea de Música en la Educación-. http://ojs.uv.es/index/php/LEEME/index ISSN: 1575-9563. Editores: Universidad de Valencia y Jesús Tejada. Visibilidad de esta revista: SCOPUS, Emerging Sources Citation Index (Clarivate), EBSCO, CINDOC (CSIC), Citefactor, COPAC, Dialnet, DICE (CSIC), DOAJ, e-revistas (CSIC), EBSCO Premier, ERIH+, Gale Cengage Learning, IN-RECS, IRESIE, LATINDEX, MIAR, OCLC Worldcat, RESH, REDIB, RILM Core Journals, SUDOC, ULRICHS. Esta revista es de acceso libre mediante licencia Creative Commons 4.0 CC by. Política de archivado: etiqueta verde SHERPA-ROMEO. 


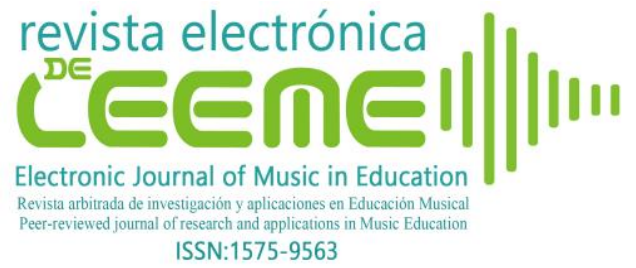

Hall, R., Atkins, L. y Fraser, J. (2014). Defining a self-evaluation digital literacy framework for secondary educators: the DigiLit Leicester project. Research in Learning Technology, 22. doi:10.3402/rlt.v22.21440

He, Y. (2020). Research on Online Teaching of Music Performance Based on Diversification and Intelligence-Take the Online Music Teaching during the COVID-19 as an Example. En 2020 International Conference on E-Commerce and Internet Technology (ECIT) (pp.193-196). Zhangjiajie: IEEE. doi.:10.1109/ECIT50008.2020.00050

Kesendere, Y., Sakin, A.Ş. y Acar, A.K. (2020). Educators' views on online/distance violin education at Covid-19 outbreak term. Journal for the Interdisciplinary Art and Education, 1(1), 1-19. Recuperado de: https://dergipark.org.tr/en/download/article$\underline{\text { file/1201556 }}$

King, A., Prior, H. y Waddington-Jones, C. (2019). Exploring teachers' and pupils' behaviour in online and face-to-face instrumental lessons, Music Education Research, 21(2), 197209. doi:10.1080/14613808.2019.1585791

Kitto, R.J. y Barnett, J. (2007). Analysis of Thin Online Interview Data. American Journal of Evaluation, 28(3), 356-368. doi:10.1177/1098214007304536

Koutsoupidou, T. (2014). Online distance learning and music training: benefits, drawbacks and challenges. Open Learning: The Journal of Open, Distance and e-Learning, 29(3), 243255. doi:10.12681/icodl.536

Krumsvik, R.J. (2014). Teacher educators' digital competence. Scandinavian Journal of Educational Research, 58(3), 269-280. doi:10.1080/00313831.2012.726273

Krumsvik, R.J. (2008). Situated learning and teachers' digital competence. Education and Information Technologies, 13(4), 279-290. doi:10.1007/s10639-008-9069-5

Kvale, S., y Brinkmann, S. (2009). Interviews: Learning the Craft of Qualitative Research Interviewing (2nd ed.). California: SAGE Publications, Inc.

Minichiello, V., Aroni, R., Timewell, E. y Alexander, L. (1990). In-Depth Interviewing: Researching people. Australia: Longman Cheshire.

Offir, B., Lev, Y. y Bezalel, R. (2008). Surface and deep learning processes in distance education: Synchronous versus asynchronous systems. Computers \& Education, 51(3), 1172-1183. doi:10.1016/j.compedu.2007.10.009 


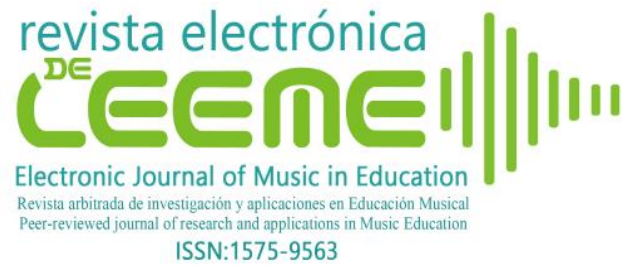

Orman, E.K. y Whitaker, J.A. (2010). Time Usage During Face-to-Face and Synchronous Distance Music Lessons. American Journal of Distance Education, 24(2), 92-103. doi:10.1080/08923641003666854

Palau, R., Usart, M. y Ucar, M.J. (2019). La competencia digital de los docentes de los conservatorios. Estudio de autopercepción en España. Revista Electrónica de LEEME, 44, 24-41. doi:10.7203/LEEME.44.15709

Prestridge, S., Tondeur, J. y Ottenbreit-Leftwich, A. (2019). Insights from ICT expert teachers about the design of educational practice: The learning opportunities of social media. Technology, Pedagogy and Education, 28(2), 157-172. doi:10.1080/1475939X.2019.1578685

Ministerio de la Presidencia, Relaciones con las Cortes y Memoria Democrática (2020). Real Decreto 463/2020, de 14 de marzo, por el que se declara el estado de alarma para la gestión de la situación de crisis sanitaria ocasionada por el COVID-19. BOE (14/03/2020), núm.67, referencia 3692, pp.25390- 25400.

Riley, P. (2009). Video-conferenced music teaching: challenges and progress. Music Education Research, 11(3), 365-375. doi:10.1080/14613800903151580

Roig, R. y García, I. (2014). Las TIC y el profesorado de música de los centros de educación secundaria de la provincia de Castellón. Didáctica, innovación y multimedia, 29, 1-14. Recuperado de: http://hdl.handle.net/10234/126245

Sailer, C., Schito, J., Kiefer, P. y Raubal, M. (2015). Teachers matter: Challenges of using a location-based mobile learning platform. En T.H. Brown, y H.J. van der Merwe (Eds.), The mobile learning voyage-From small ripples to massive open waters (pp.239-255). Berlin: Springer International Publishing.

Silverman, D. (2006). Interpreting qualitative data (3rd ed.). California: SAGE Publications Ltd.

Sims, S. (2020). School Absences and Pupil Achievement. California: Centre for Education Policy and Equalising Opportunitites, UCL Institute of Education.

Sintema, E.J. (2020). Effect of COVID-19 on the Performance of Grade 12 Students: Implications for STEM Education. EURASIA Journal of Mathematics, Science and Technology Education, 16(7), 1851. doi:10.29333/ejmste/7893

Strauss, A. y Corbin, J. (2002). Bases de la investigación cualitativa. Antioquía: Universidad de Antioquía. 


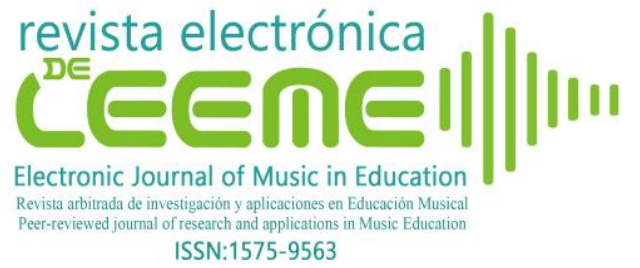
(Revista Electrónica de LEEME) Número 46, pp. 108-124 https://ojs.uv.es/index.php/LEEME/index

Teachout, D.J. (2018). The preparation of music teacher educators: a critical link. En G.E. McPherson, y G.F. Welch (Eds.), Music and Music Education in People's Lives (pp.326-329). Oxford: Oxford Handbook in Music Education.

van Gammeren, D. y Szram, A. (2019). Threshold Concepts in Online Music Education: Transforming Conservatoire Training. En European Conference on e-Learning (pp.588592). Academic Conferences International Limited. Recuperado de: https://search.proquest.com/docview/2317555249/fulltextPDF/B5343F58E5A4607PQ/1 ?accountid $=14777$

Waldron, J., Mantie, R., Partti, H. y Tobias, E.S. (2018). A brave new world: theory to practice in participatory culture and music learning and teaching. Music Education Research, 20(3), 289-304. doi:10.1080/14613808.2017.1339027

Wickramasinghe, N.C., Steele, E.J., Gorczynski, R.M., Temple, R., Tokoro, G., Wallis, D.H. y Klyce, B. (2020). Growing Evidence against Global Infection-Driven by Person-toPerson Transfer of COVID-19. VirolCurr Res, 4(1). Recuperado de: https://vixra.org/pdf/2003.0042v3.pdf

Wu, J.T., Leung, K. y Leung, G.M. (2020). Nowcasting and forecasting the potential domestic and international spread of the 2019-nCoV outbreak originating in Wuhan, China: a modelling study. The Lancet, 395(10225), 689-697. doi:10.1016/S01406736(20)30260-9

Yuen, A. y Will, W.K. (2008). Exploring teacher acceptance of e-learning technology. AsiaPacific Journal of Teacher Education, 36(3), 229-243. doi:10.1080/13598660802232779

Zhang, W., Wang, Y., Yang, L. y Wang, C. (2020). Suspending Classes Without Stopping Learning: China's Education Emergency Management Policy in the COVID-19 Outbreak. Journal of Risk and Financial Management, 13, 55. doi:10.3390/jrfm13030055 